\title{
Prenatal Diagnosis of Adrenal Neuroblastoma - Differential Diagnosis of Suprarenal Masses in the Third Trimester of Pregnancy
}

\section{Introduction}

Neuroblastoma is the most common malignant extracranial solid tumor in infancy with an incidence rate of 58 per million infants younger than one year old. It originates from the neural crest during fetal development and may arise from the sympathetic ganglia or the adrenal medulla (Gurney JG, et al. J Pediatr Hematol Oncol. 1997; 19: 428).

Prenatal detection of neuroblastoma was first described in 1983 (Fenart D, et al. Journalde Radiologie 1983; 64: 359). In the majority of antenatally diagnosed cases, neuroblastomas are adrenal in origin and right-sided. Differentiating neuroblastomas from other fetal suprarenal masses such as extralobar pulmonary sequestration, congenital adrenal hyperplasia, renal duplication, urinoma, gastric duplication cyst, and splenic cyst may be very difficult. However, improvements in ultrasound technology have made early detection possible and, combined with fetal MRI, facilitate differential diagnosis. Finally, prenatal diagnosis of a neuroblastoma is associated with a better prognosis, as it can resolve spontaneously either in utero or shortly after birth (Maki E, et al. J Ultrasound Med 2014; 33:895-904).

This is a case of a mixed cystic and solid suprarenal tumor that was identified during a routine antenatal visit in the third trimester of pregnancy and was diagnosed as an adrenal neuroblastoma.

\section{Case description}

A 27-year-old pregnant woman presented to the outpatient clinic of Alexandra Maternity Hospital in Athens, Greece during the third trimester of her pregnancy. The woman was Gravida 2 Para 0 (G2P0). The gestational age of the pregnancy was 38 weeks and 2 days. The woman's past medical history was uneventful.

During her pregnancy, she underwent prenatal testing including first trimester scan, anomaly scan, $3^{\text {rd }}$ trimester Doppler scan without any abnormal findings. During the initial ultrasound scan we detected one fetus in occiput anterior position. All growth parameters (biparietal diameter, head circumference, abdominal circumfer- ence, femur length, estimated fetal weight) and the amniotic fluid index were within normal range for the gestational age. During the scan a well circumscribed mass was detected over the left kidney ( $\triangleright$ Fig. 1). The mass appeared to have both cystic and solid areas and appeared to originate from the left adrenal gland ( $\boldsymbol{F i g . ~ 1 ) . ~ T h e ~ a n a t o m y ~}$ of the left kidney was not altered. The mass measured $4.40 \times 4.12 \mathrm{~cm}$ ( $>$ Fig. 2). Doppler evaluation did not reveal increased blood flow towards the mass nor a single feeding vessel ( $\triangleright$ Fig. 3 ). The right kidney and right adrenal gland appeared normal ( $\vee$ Fig. 4a and b). Extensive ultrasound examination of the fetus revealed no other abnormalities. After careful evaluation of the ultrasound appearance of the tumor, the diagnosis of adrenal neuroblastoma was suggested.

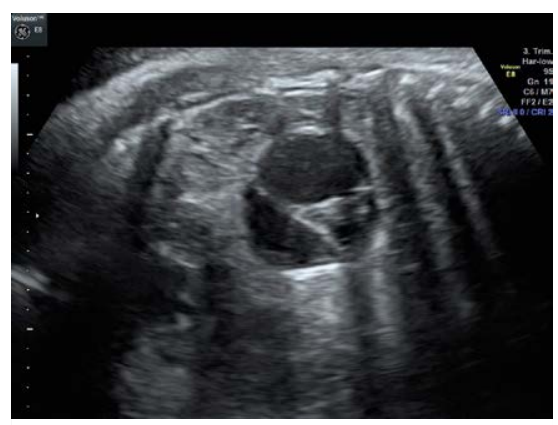

Fig. 1 The mass is well circumscribed with both cystic and solid areas and appears to originate from the left adrenal gland.

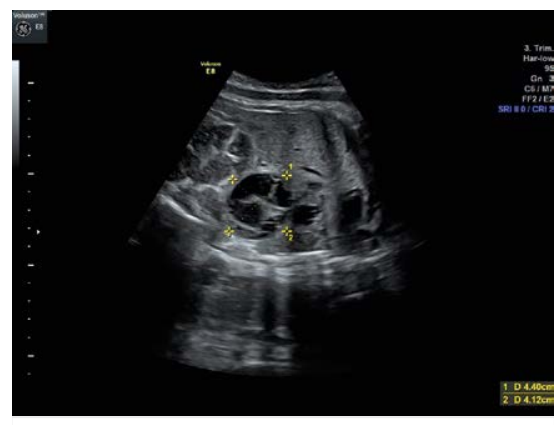

Fig. 2 The mass measured $4.4 \times$ $4.12 \mathrm{~cm}$ at 38 weeks and 2 days of pregnancy.

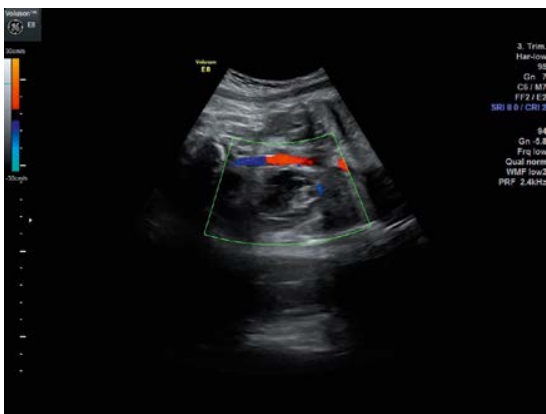

- Fig. 3 Doppler evaluation did not reveal increased blood flow towards the mass or a single feeding vessel.

With regards to the gestational age of the pregnancy, induction of labor was scheduled at $39^{+1}$ weeks, resulting in the birth of a male neonate with APGAR score 9 in the first minute. Transabdominal ultrasound examination corroborated the diagnosis. The neonate was transported to the pediatric oncology department where it underwent abdominal CT scan (computed tomography scan), serum NSE (neuron specific enolase) and urine VMA (vanillylmandelic acid) and HVA (homovanillic acid) tests. The CT scan, in accordance with the prenatal ultrasound findings, revealed a well circumscribed mass above the left kidney ( $\mathbf{F i g . ~ 5 ) . ~ N S E ~ w a s ~}$ $65.3 \mathrm{mg} / \mathrm{l}$ (normal value $<12.5 \mathrm{mg} / \mathrm{l}$ ), urine VMA was $63.2 \mathrm{mg} / \mathrm{g}$ creatinine (cr) (normal value $<27 \mathrm{mg} / \mathrm{g} \mathrm{cr}$ ) and urine HVA was $47.3 \mathrm{mg} / \mathrm{g} \mathrm{cr}$ (normal value < $35.0 \mathrm{mg} / \mathrm{g} \mathrm{cr}$ ) (LaBrosse EH et al. Cancer Res. 1980; 40: 1995-2001). The CT scan and elevated serum and urine markers confirmed the diagnosis of neuroblastoma. A strategy of observation with close follow-up visits was adopted, as prenatally diagnosed localized neuroblastomas (stage I) often regress spontaneously (Nuchtern JG et al. Ann Surg. 2012; 256: 573.).

\section{Discussion}

Antenatal detection and differential diagnosis of a suprarenal mass are challenging and essential for prenatal counseling, management of the pregnancy, delivery planning and further management and prognosis of the neonate. Fetal MRI is very helpful 

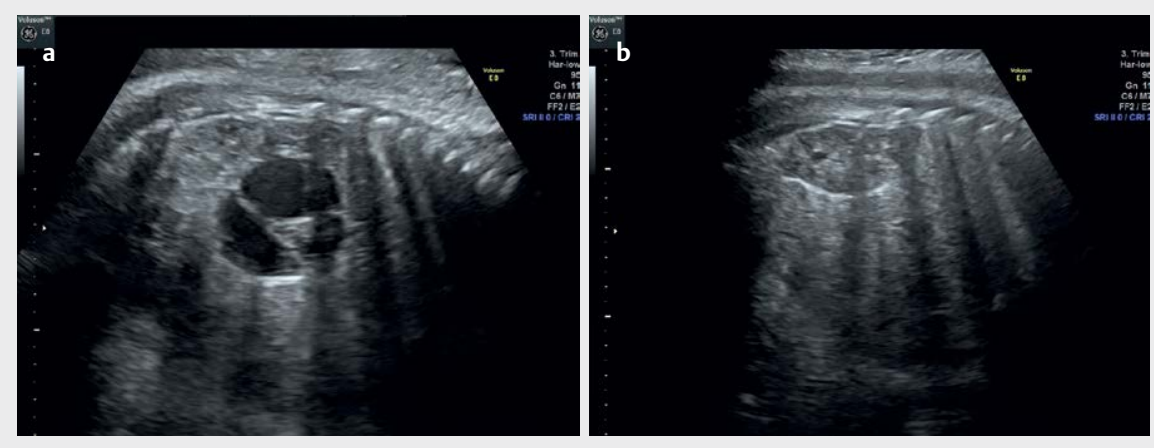

- Fig. 4 The right kidney and right adrenal gland appear normal.

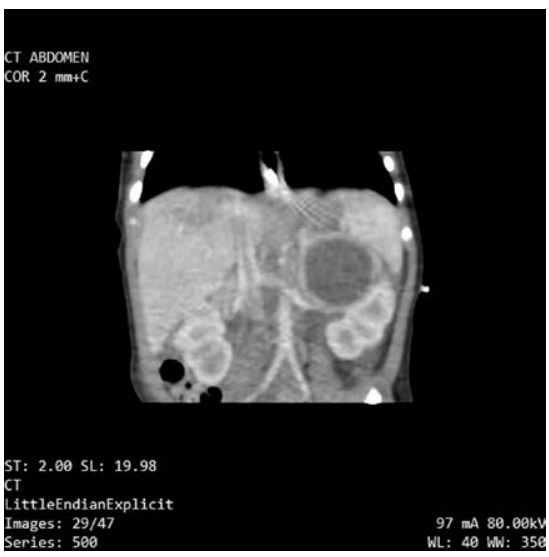

- Fig. 5 Abdominal CT scan confirmed the diagnosis of neuroblastoma of the left adrenal gland.

when the gestational age of the pregnancy is not as advanced as in our case.

Fetal neuroblastoma is the most common non-hematologic malignant tumor in infants, but it is difficult to diagnose, as its ultrasound characteristics may vary between cystic, mixed solid and cystic and completely solid with or without calcifications. The cystic features of the tumor are an important prognostic factor, as cystic neuroblastomas have a better prognosis, compared to non-cystic (Isaacs $\mathrm{H}$, et al. Fetal and Pediatr Pathology. 2007; 26: 177184). It should be carefully differentiated from other suprarenal masses, including extralobar pulmonary sequestration, congenital adrenal hyperplasia, renal duplication, urinoma, gastric duplication cyst, and splenic cyst. Differential diagnosis is mostly based on the ultrasound characteristics of the tumor, position, invasion of nearby tissues, blood perfusion and the gestation- al age of the pregnancy. When a positive diagnosis cannot be made based solely on the ultrasound examination, fetal MRI should be considered.

Extralobar pulmonary sequestrations are more common than neuroblastomas and $10-15 \%$ are subdiaphragmatic (Maki E, et al. JUltrasound Med 2014; 33: 895-904). Hence, differentiating subdiaphragmatic extralobar pulmonary sequestrations from adrenal neuroblastomas is very important. Neuroblastomas usually appear as cystic masses on the right side and in the majority of cases they are detected during the third trimester. On the other hand, extralobar pulmonary sequestrations, are echogenic, mainly left-sided and are usually detectable in the second trimester (Daltro PA, et al. Pediatric Chest Imaging Heidelberg: Springer-Verlag. 2008; 397-416). Another major difference is the lack of a single feeding artery in neuroblastomas, since they mostly have only peripheral blood flow or appear to have no flow at all. Congenital adrenal hyperplasia is mostly bilateral, with the left adrenal gland being asymmetrically larger than the right and a large feeding artery being visible in Doppler flow. Adrenal hemorrhage and ambiguous genitalia may coexist and facilitate the diagnosis. Urinomas encapsulated in the Gerota facia may have septations and poor perfusion, hence mimicking the presence of an adrenal cystic neuroblastoma. Dilation of the renal pelvis and the ureter may help with the differential diagnosis. Splenic cysts are usually anechoic and contained within the spleen. Finally, gastric duplication cysts may appear as suprarenal masses but they are easily recognized from the three layer wall and the presence of peristalsis (Maki E, et al. JUltrasound Med 2014; 33: 895-904).

In our case, the diagnosis of the fetal neuroblastoma was especially difficult since, unlike the majority of cases, it was left-sided, its appearance was complex (mixed cystic and solid) and the gestational age of the pregnancy was very advanced.

Neuroblastoma treatment requires a multidisciplinary approach and the treatment strategies, including surgical resection, chemotherapy or expectant management with ultrasonographic follow-up, are based on the staging and classification of the tumor. It is widely accepted that accurate prenatal diagnosis is crucial for the prenatal and postnatal management of neuroblastomas, as early detection provides important information for the delivery and parental counseling while at the same time allowing for the immediate management of this common malignant tumor, thereby improving the outcome of the young patients.

\section{Conflict of Interest}

The authors declare that they have no conflict of interest.

Authors

Alexandros Psarris' ${ }^{\mathbb{D}}$, Michail Sindos², Anastasia Dimopoulou ${ }^{3}$, Panagiotis Antsaklis ${ }^{1}$, Antonios Psarakis ${ }^{1}$, Thomas Kataras ${ }^{1}$, Dimitrios Loutradis ${ }^{2}$, Georgios Daskalakis ${ }^{4}$ 
1 First Department of Obstetrics and Gynecology, National and Kapodistrian University of Athens, Athens, Greece

2 Athens University, 1st Dept of Obstet Gynecology, Athens, Greece

3 Department of Paediatric Surgery, National and Kapodistrian University of Athens, Attikon Hospital, Athens, Greece

4 First Department of Obstetrics and Gynecology, University of Athens, Greece, Athens, Greece

\section{Correspondence}

Dr. Alexandros Psarris

First Department of Obstetrics and

Gynecology, National and Kapodistrian

University of Athens,

V. Sofias 80 and Lourou Street

11528 Athens

Greece

Tel : 6979232977

psarris.alexandros@gmail.com
DOI https://doi.org/10.1055/a-1070-8651

Ultrasound Int Open 2019; 5: E93-E96

(c) Georg Thieme Verlag KG Stuttgart · New

York

ISSN 2199-7152

() (1) $\odot \ominus$ 